\title{
Análisis de la relación existente entre los tipos de motivación y la intención de ser físicamente activo en Educación Física de Primaria
}

\section{Pedro Muñoz Sánchez}

\section{Graduado en Educación de Primaria, con Mención en EF (Universidad de Huelva) \\ Email: pedrixy 10 1994@hotmail.com}

\section{RESUMEN:}

El objetivo fundamental de la investigación fue describir y correlacionar los distintos tipos de motivación con la intención de ser físicamente activo. La muestra utilizada fue de 85 alumnos y alumnas de Educación Primaria con una media de edad de 10.94 años. En las clases de Educación Física se midió la motivación y la intención de ser físicamente activos. Los resultados de los estadísticos descriptivos y de las correlaciones mostraron que los tipos de motivación más autodeterminados tenían una relación positiva con la intención de ser físicamente activos. En la discusión se hace una interpretación de los resultados relacionándolos con la teoría recogida y mencionando que el docente tiene un papel importante en la motivación del alumno en la clase de Educación Física, a la vez que se dan recomendaciones para que la motivación que se genere tenga consecuencias positivas.

PALABRAS CLAVE: Teoría de la autodeterminación, Motivación intrínseca, Educación Primaria, Desmotivación, Adherencia a la práctica deportiva.

\section{Analysis of the existing relation between the types of motivation and the intention to be physically active in primary physical education}

\section{ABSTRACT:}

The main goal of the research was to describe and relate the different types of motivation with the intention of being physically active. The sample used was 85 primary school pupils with a mean age of 10.94 years. Physical Education classes measured motivation and intention to be physically active. The results of descriptive statistics and correlations showed that the most self-determined types of motivation had a positive relationship with the intention to be physically active. In the study an interpretation of the results by relating them to the collected theory and mentioning that the teacher has an important role in the motivation of the student in the Physical Education class, while giving recommendations so that the motivation that is generated is going to have positive consequences.

KEY WORDS: Self-determination theory, Intrinsic motivation, Primary education, Amotivation, Adherence to sports practice. 


\section{INTRODUCCIÓN}

El problema que se va a estudiar en este trabajo es la influencia que tiene la motivación en las clases de Educación Física sobre la intención de ser físicamente activo en un centro de la provincia de Huelva.

Según Moreno, Moreno y Cervelló (2007), la práctica físico-deportiva es importante en el desarrollo de las personas, ya que ayuda a prevenir enfermedades que afectan al campo físico, como por ejemplo la obesidad, y al campo psicológico, como pueden ser la ansiedad y la depresión; por lo que es importante que los niños, desde pequeños, adquieran hábitos de este tipo y que continúen teniéndolos a lo largo de toda su vida. Siguiendo con lo anteriormente enunciado, hay estudios en los que se afirma que la práctica físico-deportiva es un medio que ayuda a eliminar factores de riesgo arterial y cardiovascular. Por otro lado, también influye en el desarrollo y fortalecimiento de los sistemas óseo y muscular y en la autonomía y el autoconcepto. Sin embargo, aunque la actividad física tenga todos estos beneficios, existe un gran número de personas sedentarias.

En el año 2015 en España, el 46,5\% de la población mayor de 14 años no practicó deporte (Ministerio de Educación, Cultura y Deporte, 2015), lo cual supone un poco menos de la mitad de la población del país, dato bastante alto que aumenta a la vez que la edad de las personas crece. Este dato es preocupante, ya que casi la mitad del país no practicó deporte (no fue físicamente activo), aspecto muy importante para la salud. Además, Cecchini, Fernández-Losa, González, Fernández y Méndez (2012) enuncian que hay un importante número de adolescentes y jóvenes que abandonan la práctica físico-deportiva, aumentando el abandono conforme va aumentando la edad de los sujetos.

En busca de una solución a este problema, Cuevas, Contreras, Fernández y González-Martí (2014) exponen que una de las claves de la intención de ser físicamente activo puede tener relación con el tipo de motivación que las personas reciben en la etapa escolar. Una forma de motivar es, a partir de la teoría de la autodeterminación, la producida por el placer de participar, la diversión y la satisfacción de los individuos, es decir, que en ella se fomenta la motivación intrínseca, la cual tiene una alta capacidad predictiva para la intención de ser físicamente activo.

Hablando de este mismo aspecto, Martínez, Alonso, González-Cutre, Parra y Moreno (2010) y Moreno y Cervelló (2003) decían que la motivación es un elemento muy importante en el proceso de enseñanza-aprendizaje, afirmando que un alumno motivado en la clase de educación física siente placer y tiene interés por ejercitarse y aprender en ella.

Cera, Almagro, Conde y Sáenz-López (2015) hablan de la motivación como un concepto abstracto, multidimensional y explicativo de la conducta humana. Además, dan una definición en la que la consideran como un mecanismo psicológico que controla la dirección, intensidad y persistencia de la conducta. En ella, la dirección son las metas que una persona quiere conseguir, la intensidad es el esfuerzo que se hace para lograrlas y la persistencia es el tiempo que se emplea en la consecución de las mismas.

Por otro lado, según Moreno y Cervelló (2003), el comportamiento del docente también es importante en la creación de actitudes positivas hacia la educación física además de la metodología utilizada por el mismo, ya que aquellas que favorecen la 
autonomía y la toma de decisiones por parte del alumnado, proporcionan una mayor satisfacción, interés y mayores niveles de práctica extraescolar. También es importante el hecho de que el alumnado le da mayor importancia a la Educación Física cuando el profesor tiene una actitud democrática, comprensiva, buena, alegre, justa, agradable y acepta la iniciativa individual de los alumnos.

Siguiendo la teoría de la autodeterminación, Sáenz-López, Castillo y Conde (2009) y Méndez-Giménez, Fernández-Río y Cecchini-Estrada (2013) afirman que esta teoría trata de analizar cómo determinados factores sociales influyen en la satisfacción de las necesidades psicológicas básicas de autonomía, competencia y relación con los demás, que parecen ser principales para el crecimiento y la integración, así como para el desarrollo social y el bienestar personal. González-Cutre, Martínez, Gómez y Moreno (2010) y Méndez-Giménez, et al. (2013) explican estas tres necesidades, de modo que, en cuanto a la necesidad de autonomía, ésta se refiere al esfuerzo que hacen las personas para sentirse el origen de sus hechos y tener influencia para determinar su propio comportamiento. La necesidad de sentirse capaz o competente se basa en intentar controlar el resultado y experimentar ser eficaz. Por otro lado, la necesidad de relacionarse con los demás se refiere al esfuerzo por interactuar y mostrar interés por otros, además de sentir que los demás tienen una relación auténtica o verdadera contigo y experimentar satisfacción con la sociedad. Esta necesidad está determinada por dos factores, sentirse aceptado e intimar con los demás.

Como afirman Méndez-Giménez, et al. (2013) y González-Cutre, et al. (2010), las tres necesidades enunciadas en la teoría de la autodeterminación influirán en la motivación de forma que, al aumentar la percepción de competencia, la autonomía y la relación con los demás, se experimentará una motivación intrínseca, mientras que la disminución de las mismas se asociará con una menor motivación intrínseca, una mayor motivación extrínseca y una mayor desmotivación. Almagro, Navarro, Paramio y Sáenz-López (2015) enuncian que la teoría de la autodeterminación establece distintos tipos de motivación de forma continua dependiendo del nivel de autodeterminación. De esta forma, de menor a mayor autodeterminación, están la desmotivación, la motivación extrínseca (regulación externa, regulación identificada, regulación introyectada y regulación integrada) y la motivación intrínseca.

Según González-Cutre, et al. (2010), González-Cutre, Sicilia y Moreno (2011), Almagro, et al. (2015) y Cecchini, et al. (2012), la motivación es intrínseca cuando genera un compromiso de un sujeto con una actividad por el placer, la curiosidad y disfrute que le genera, lo que hace que la actividad sea un fin por sí misma. Esta motivación intrínseca se mantiene con la competencia del sujeto y sus creencias de ser eficaz, por tanto, el discente participará en las actividades por la satisfacción y el placer propio de la actividad, de forma que si una persona se siente capaz y eficaz incrementará su motivación intrínseca y, por consiguiente, su intención de ser físicamente activo (Almagro, Sáenz-López, González-Cutre, y Moreno, 2011; Moreno y Martínez, 2006). Lo cual coincide con lo enunciado por Cuevas et al. (2014), los cuales dicen que la motivación intrínseca tiene una gran capacidad para predecir la intención de ser físicamente activo de los alumnos. Por el contrario, en la motivación extrínseca no se actúa por el placer que le genera en si misma la actividad, sino por el placer externo de la misma, es decir, está determinada por recompensas o factores externos. Finalmente, la desmotivación se basa en que el sujeto no tiene intención de hacer algo, haciendo que la actividad no se valore, no sea organizada y acompañada con sentimientos de miedo, frustración o depresión, haciendo que el alumno no se sienta 
competente para hacerla o no espere conseguir el resultado buscado (González-Cutre, et al., 2010; Almagro, et al., 2015; Moreno y Martínez, 2006).

Por tanto, utilizando el marco teórico expuesto anteriormente, el objetivo de este estudio fue describir y correlacionar los distintos tipos de motivación con la intención de ser físicamente activo. Al mismo tiempo, siguiendo lo establecido por el marco teórico recogido anteriormente, se planteó la siguiente hipótesis: la motivación más autodeterminada se relacionará positivamente con la intención de ser físicamente activo, mientras que la motivación menos autodeterminada se relacionará de forma negativa con la intención de ser físicamente activo.

\section{MÉTODO}

\subsection{Participantes}

El estudio estuvo compuesto por una muestra total de 85 alumnos y alumnas de la materia de Educación Física, de los cuales 42 eran alumnos y 43 eran alumnas, las edades oscilaban entre 10 y 12 años, siendo la edad media de 10.94 años $(D T=$ .64). El muestreo fue intencional (por la accesibilidad del centro educativo incluido en el estudio). El alumnado pertenecía a un CEIP de la provincia de Huelva, los cuales cursaban los cursos de $5^{\circ}$ y $6^{\circ}$ de Primaria. Los alumnos participantes hacían Educación Física dos días a la semana durante cuarenta y cinco minutos cada día.

La participación en este estudio por parte de los alumnos fue voluntaria, contestando los cuestionarios durante las clases de Educación Física en presencia en todo momento de su maestro de EF y por el autor de este artículo, por si tenían alguna duda. El tiempo que tardaron en rellenar el cuestionario fue entre 15 y 20 minutos.

\subsection{Instrumentos}

Los alumnos que participaron en el estudio tuvieron que rellenar un cuestionario que incluía datos socio-demográficos (género, curso, edad, etc.) y las siguientes escalas:

Escala del Locus Percibido de Causalidad en Educación Física (PLOC). Se utilizó la versión testada en el contexto español por Moreno, González-Cutre y Chillón (2009) de la Perceived Locus of Causality Scale (PLOC) de Goudas, Biddle y Fox (1994). La sentencia previa a los ítems fue "Participo en las clases de Educación Física". La escala estaba compuesta por 24 ítems agrupados en cuatro dimensiones, compuesta cada una por cuatro ítems. Las dimensiones son la Motivación Intrínseca (e.g. "Porque la educación física es divertida"), Regulación Identificada (e.g. "Porque quiero aprender habilidades deportivas"), Regulación Introyectada (e.g. "Porque me sentiría mal conmigo mismo si no lo hiciera"), Regulación Externa (e.g. "Porque tendré problemas si no lo hago") y Desmotivación (e.g. "Pero no comprendo porque debemos tener educación física"). Las respuestas fueron puntuadas con una escala tipo Likert, con un rango de puntuación que oscilaba entre 1 (Totalmente en desacuerdo) y 7 (Totalmente de acuerdo). Se obtuvieron valores alfa de Cronbach .46 para la motivación intrínseca, .48 para la regulación identificada, .57 para la regulación introyectada, .77 para la regulación externa y .59 para la desmotivación. 
Medida de la Intencionalidad para ser Físicamente Activo (MIFA). Se utilizó la versión adaptada y traducida al español (Moreno, Moreno, y Cervelló, 2007) de la Intention to be Physically Active Scale (IPAS) de Hein, Müür y Koka (2004).La escala estaba encabezada por la sentencia "Respecto a tu intención de practicar alguna actividad físico-deportiva...". Además, se modificaron dos ítems para que pudiese ser empleado en Primaria y Secundaria (en los ítems 3 y 4 , se eliminó el comienzo del ítem: "Después de terminar el instituto,"). La Escala estaba compuesta por cinco ítems para medir la intención de ser físicamente activo (e.g. "Habitualmente practico deporte en mi tiempo libre"). Las respuestas corresponden a una escala tipo Likert que oscila de 1 (Totalmente en desacuerdo) a 5 (Totalmente de acuerdo). El alfa de Cronbach fue de .54.

\subsection{Procedimiento}

Para poder realizar la recogida de datos, se contactó con el Centro de Educación Primaria para solicitar la correspondiente autorización y ayuda de los docentes para que fuese más fácil la recogida de datos.

Mediante una autorización escrita, se solicitó a los padres el permiso para que sus hijos e hijas, si él o ella lo deseaba, participara en el estudio, dando la información de que a sus hijos e hijas se les pediría que cumplimentasen un cuestionario durante la clase de educación física. Además, se garantizó el anonimato de los participantes en dicho estudio.

Finalmente, los cuestionarios se administraron con la presencia de un investigador para poder resolver cualquier duda que pudieran tener los discentes y concienciándolos de la importancia que tenía rellenar los cuestionarios sinceramente.

\subsection{Análisis de datos}

En primer lugar, se hizo una depuración de la matriz de datos y se realizaron los análisis necesarios para garantizar la fiabilidad de las escalas utilizadas en el estudio. A continuación, se hizo el cálculo de los estadísticos descriptivos de las distintas variables de estudio y las correlaciones bivariadas. Los análisis se realizaron con los paquetes estadísticos SPSS 22.0.

\section{RESULTADOS}

En la Tabla 1, se exponen los estadísticos descriptivos (media y desviación típica) y las correlaciones bivariadas entre las variables estudiadas.

En lo referido a las puntuaciones medias obtenidas en cada variable, el valor más alto lo obtuvo la motivación intrínseca y, a continuación, la regulación identificada. En general, los resultados obtenidos fueron que las formas motivacionales más autodeterminadas adquirieron puntuaciones medias más altas que las menos autodeterminadas, las cuales son la regulación introyectada, externa y la desmotivación. Al mismo tiempo, la intención obtuvo una media alta.

El análisis de correlación mostró que la motivación intrínseca se correlacionaba de forma positiva y estadísticamente significativa con la regulación identificada y con la intención de ser físicamente activo, mientras que con la desmotivación tenía una 
correlación negativa y estadísticamente significativa. La regulación identificada correlacionó de forma positiva y estadísticamente significativa con la motivación intrínseca y la intención de ser físicamente activo. Por otro lado, la regulación introyectada correlacionó de forma positiva y estadísticamente significativa con la regulación externa, la desmotivación y la intención de ser físicamente activo. La regulación externa se correlacionó positivamente y de forma significativa con la regulación introyectada y la desmotivación. La desmotivación se relacionó negativamente y significativamente con la motivación intrínseca; sin embargo, lo hizo positivamente con la regulación introyectada y externa. La intención de ser físicamente activo se correlacionó positivamente y significativamente con la motivación intrínseca, la regulación identificada y la introyectada.

Tabla 1. Estadísticos descriptivos y correlaciones de las variables

\begin{tabular}{lcccccccc}
\hline \multicolumn{1}{c}{ Variables } & $\boldsymbol{M}$ & $\boldsymbol{D T}$ & $\mathbf{1}$ & $\mathbf{2}$ & $\mathbf{3}$ & $\mathbf{4}$ & $\mathbf{5}$ & $\mathbf{6}$ \\
\hline 1. MI & 6.53 & .64 & - & $.44^{\star *}$ & .215 & .04 & $-.24^{*}$ & $.42^{* *}$ \\
2. R. Identificada & 6.43 & .67 & & - & .11 & .04 & -.11 & $.49^{* *}$ \\
3. R. Introyectada & 4.41 & 1.46 & & & - & $.55^{\star *}$ & $.37^{\star *}$ & $.28^{* *}$ \\
4. R. Externa & 3.23 & 1.64 & & & & - & $.38^{* *}$ & .12 \\
5. Desmotivación & 1.87 & 1.15 & & & & & - & .01 \\
6. Intención & 4.50 & .56 & & & & & & -
\end{tabular}

Nota. ${ }^{* *} \mathrm{p}<.01 ;{ }^{*} \mathrm{p}<.05 ; M=$ Media; $D T=$ Desviación típica; $\mathrm{Ml}=$ Motivación intrínseca; $\mathrm{R} .=$ Regulación; EF = Educación Física.

\section{DISCUSIÓN}

Este estudio ha buscado describir y correlacionar los distintos tipos de motivación con la intención de ser físicamente activo. Hay trabajos que han analizado las relaciones mostradas en esta investigación. Estudios anteriores (Almagro, et al., 2011; Cuevas, et al., 2014; González-Cutre, et al., 2010; Almagro, Navarro, et al., 2015; Cechini, et al., 2012) ya han enunciado consecuencias positivas de los tipos de motivación más autodeterminadas en clase de Educación Física sobre la intención de ser físicamente activo. Por otro lado, relación positiva entre una de las motivaciones no tan autodeterminadas como es la regulación introyectada y la intención de ser físicamente activo, esta relación no se ha encontrado en ninguno de los estudios revisados.

Los resultados de las correlaciones bivariadas y de los estadísticos descriptivos de este estudio coincidieron con lo encontrado en otros estudios (Sáenz-López, et al., 2009; González-Cutre, et al., 2010; Almagro, et al., 2015; Cecchini, et al., 2012; Méndez-Giménez, et al., 2013), ya que las motivaciones más autodeterminadas (motivación intrínseca y regulación identificada) tuvieron las puntuaciones medias más altas que las menos autodeterminadas (regulación introyectada, externa y desmotivación). Al mismo tiempo, la intención de ser físicamente activo obtuvo una media alta. Por otro lado, las correlaciones entre las variables siguieron la línea de la hipótesis de la investigación, la motivación más autodeterminada se correlacionó de forma positiva con la intención de ser físicamente activo. Sin embargo, una de las correlaciones no siguió la línea establecida por la hipótesis, ya que en ella se establecía una relación positiva entre la regulación introyectada y la intención de ser 
físicamente activo (aunque en menor grado que con las motivaciones más autodeterminadas).

Esta investigación muestra que la motivación intrínseca y la regulación identificada se asocian positivamente con la intención de ser físicamente activo, coincidiendo con lo encontrado por Cuevas et al. (2014) y Almagro et al. (2011). Por otro lado, el aumento de la motivación intrínseca se correlacionó negativamente con la desmotivación, es decir, que al descender de la motivación intrínseca aumentará la desmotivación y viceversa, coincidiendo con lo enunciado por González-Cutre et al. (2010) y con lo establecido en la hipótesis de la investigación. Para terminar con las correlaciones, se encontró una relación positiva entre los tipos de motivación menos autodeterminadas (regulación introyectada, externa y la desmotivación). Además, teniendo en cuenta que la correlación positiva entre la motivación intrínseca y la regulación identifica y la relación negativa entre las motivación intrínseca y la desmotivación, se puede decir que el descenso de la motivación más autodeterminada (motivación intrínseca y regulación identificada) puede implicar un aumento de la motivación menos autodeterminada (regulación introyectada, externa y desmotivación), aspecto que es recogido por González-Cutre et al. (2010). Es obvio que un alumno que participe en las clases de Educación Física por la satisfacción propia y el placer de realizar las actividades que se propongan (lo cual promueve una motivación autodeterminada), tendrá una mayor predisposición por ser físicamente activo, ya que seguirá realizando actividad física por los mismos motivos por los que participa en la clase de Educación Física.

Concluyendo, este estudio muestra la importancia que tiene la motivación en los discentes en las clases de Educación Física, ya que esta influye de forma directa en la intención de ser físicamente activos en un futuro o en su tiempo libre. Por tanto, esta investigación, demuestra que, como ya dijeron González-Cutre et al. (2010), si se satisfacen las tres necesidades enunciadas en la teoría de la autodeterminación (competencia, autonomía y relación con los demás), aumentará la motivación intrínseca, la cual según diferentes autores (Almagro et al., 2011; Cuevas et al., 2014; Moreno y Martínez, 2006) es un predictor de la intención de ser físicamente activo. Mientras que, si por el contrario, no se satisfacen esas necesidades, aumentará la motivación extrínseca y la desmotivación, provocando a su vez un descenso de la motivación intrínseca y, por consiguiente, un descenso de la intención de ser físicamente activo. En definitiva, es muy importante satisfacer las necesidades psicológicas básicas para mejorar la motivación del alumnado y la intención de ser físicamente activos. Esta satisfacción dependerá en gran medida del docente y del clima motivacional que este emplee en el aula.

A continuación, con la intención de ayudar a los profesores de Educación Física que busquen mejorar el clima motivacional en sus clases, se van a exponer algunas recomendaciones para conseguir satisfacer las tres necesidades enunciadas en la teoría de la autodeterminación y, de esta manera, aumentar la motivación autodeterminada. Estas recomendaciones están basadas en trabajos anteriores (Sáenz-López, et al., 2009; González-Cutre, et al., 2011; Moreno y Martínez, 2006) y son las siguientes:

- Aprender jugando: las propuestas deben ser a través del juego, porque resulta más motivante para el alumnado que las actividades o ejercicios analíticos.

- Tener una actitud de paciencia y clima favorable: trabajo en grupo, sin obligar a participar, sino animar a que lo hagan para que no se sientan incómodos con contenidos que puede que no hayan trabajado antes. 
- Utilizar recursos motivacionales: la situación del docente, participación del mismo con los alumnos en la clase y utilizar artefactos como maquillaje, disfraces, música, etc.

- Proponer tareas variadas: que las actividades no sean siempre las mismas.

- Hacer partícipe a los discentes en la toma de decisiones y en la evaluación.

- Hacer grupos variados (no siempre los mismos) y seguir distintos criterios de agrupación, es decir, realizar agrupaciones de forma flexible y heterogénea.

- Evaluar el progreso de forma individualizada, privada, significativa y evitando comparaciones entre alumnos.

- Disponer del tiempo necesario para la práctica y la mejora de las tareas, respetando el tiempo necesario para realizar cada actividad, teniendo en cuenta la práctica y la edad de los alumnos y posibilitando los tiempos de aprendizaje.

- Variedad en el material: que el material utilizado no sea siempre el mismo.

- Plantear juegos en los que se den situaciones de cooperación y oposición.

- Que sean actividades significativas, es decir, que les sirva para obtener aprendizajes a largo plazo.

- Hacer comunicaciones positivas acerca de los resultados, es decir, utilizar el refuerzo o feedback positivo.

- Dar a todos los discentes la misma posibilidad de liderazgo.

- Dar recompensas y evitar el castigo. Al mismo tiempo, acordar con el alumnado desde el principio de curso las recompensas y castigos.

- Favorecer las relaciones entre los alumnos.

En conclusión, el docente es una pieza importante en la mejora de la motivación del alumnado en las clases de educación física, aunque, como recogen Almagro et al. (2011), también influyen otros factores como son padres, amigos, entrenadores, etc. Además, aplicando estas recomendaciones, es probable que la motivación del alumnado en la clase de Educación Física mejore y, con ella, la intención de ser físicamente activo.

Para terminar, cabe comentar que esta investigación o estudio presenta limitaciones, ya que la muestra obtenida no es muy grande y que el alfa de Cronbach para algunas variables no es el adecuado $(\alpha \geq .70)$, por lo que habrá que tomar los resultados de este estudio con precaución. Este estudio ha tratado la posible relación entre los distintos tipos de motivación y la intención de ser físicamente activo. De esta forma, la investigación debe ser tomada como exploratoria, ya que ayuda a comprender mejor los tipos de motivación que se experimentan en Educación Física y su repercusión positiva o negativa en la intención de ser físicamente activo en el futuro. Además, los datos obtenidos podrían ayudar a abordar nuevas formas de impartir Educación Física, para que ésta ayude a conseguir un mayor número de alumnos con intención de ser físicamente activos en un futuro o en su tiempo libre. Sin embargo, con este estudio no sería suficiente, siendo necesarias otras investigaciones en las cuales apoyarse. 


\section{CONCLUSIONES}

Generar una motivación intrínseca o más autodeterminada en los alumnos en las clases de Educación física es importante en la predicción de la intención de ser físicamente activos durante su tiempo libre o en un futuro. Por tanto, es importante que el docente de Educación Física utilice estrategias para mejorar la motivación más autodeterminada en el alumno, y con ella, los distintos factores positivos que ello conlleva.

\section{REFERENCIAS}

Almagro, B. J., Navarro, I., Paramio, G., y Sáenz-López, P. (2015). Consecuencias de la motivación en las clases de Educación Física. EmásF: Revista Digital de Educación Física, 34, 26-41. Recuperado de http://emasf.webcindario.com/Consecuencias de la motivacion en las clases de EF.pdf

Almagro, B. J., Sáenz-López, P., González-Cutre, D., y Moreno, J. A. (2011). Clima motivacional percibido, necesidades psicológicas y motivación intrínseca como predictores del compromiso deportivo en adolescentes. Revista Internacional de Ciencias del Deporte, 7(25), 250-265. Recuperado de http://www.cafyd.com/REVISTA/02501.pdf

Cecchini, J. A., Fernández-Losa, J. L., González, C., Fernández, J., y Méndez, A. (2012). La caída de la motivación autodeterminada en jóvenes escolares. SPORT TK: Revista Euroamericana de Ciencias del Deporte, 1(1), 25-31. Recuperado de http://revistas.um.es/sportk/article/view/185531/153601

Cera, E., Almagro, B. J., Conde, C., y Sáenz-López, P. (2015). Inteligencia emocional y motivación en educación física en secundaria. Retos, 27, 8-13. Recuperado de http://retos.org/numero 27/8-13.pdf

Cuevas, R., Contreras, O., Fernández, J. G., y González-Martí, I. (2014). Influencia de la motivación y el autoconcepto físico sobre la intención de ser físicamente activo. Revista Mexicana de Psicología, 31(1), 17-24. Recuperado de http://www.redalyc.org/html/2430/243033030003/

González-Cutre, D., Martínez, A., Gómez, A., y Moreno, J. A. (2010). La motivación autodeterminada en la actividad física y el deporte: Conceptualización. En J. A. Moreno y E. Cervelló (Coords.), Motivación en la actividad física y el deporte. (pp. 119-150) Sevilla: Editorial Wanceulen.

González-Cutre, D., Sicilia, A., y Moreno, J. A. (2011). Un estudio cuasi-experimental de los efectos del clima motivador tarea en las clases de Educación Física. Revista de Educación. 356, 677-700. Recuperado de http://www.mecd.gob.es/dctm/revista-deeducacion/articulosre356/re35628.pdf?documentld=0901e72b81203178

Martínez, C., Alonso, N., González-Cutre, D., Parra, N., y Moreno, J. A. (2010). Las metas de logro y sociales y sociales como mecanismo de motivación en la práctica físico-deportiva: Conceptualización. En J. A. Moreno y E. Cervelló 
(Coords.), Motivación en la actividad física y el deporte. (pp. 41-68) Sevilla: Editorial Wanceulen.

Méndez-Giménez, A., Fernández-Río, J., y Cecchini-Estrada, J. A. (2013). Climas motivacionales, necesidades, motivación y resultados en Educación Física. Aula Abierta, 41(1), 63-72. Recuperado de https://www.researchgate.net/profile/Antonio Mendez-

Gimenez/publication/235941097 Climas motivacionales necesidades motivac ion y resultados en Educacion Fisica/links/09e415148988d7984a000000.pdf

Moreno, J. A., y Cervelló, E. (2003). Pensamiento del alumno hacia la educación física: su relación con la práctica deportiva y el carácter del educador. Enseñanza \& Teaching: Revista Interuniversitaria de Didáctica, 21, 345-362. Recuperado de http://e-

spacio.uned.es/fez/eserv.php?pid=bibliuned:20350\&dsID=pensamiento alumno . pdf

Moreno, J. A., González-Cutre, D., y Chillón, M. (2009). Preliminary validation in Spanish of a scale designed to measure motivation in physical education classes: the Perceived Locus of Causality (PLOC) Scale. The Spanish Journal of Psychology, 12(1), 327-337.

Moreno, J. A., y Martínez, A. (2006). Importancia de la teoría de la autodeterminación en la práctica física-deportiva: fundamentos e implicaciones prácticas. Cuadernos de Psicología del Deporte, 6(2), 39-54. Recuperado de http://revistas.um.es/cpd/article/view/113871/107881

Moreno, J. A., Moreno, R., y Cervelló, E. (2007). El autoconcepto físico como predictor de la intención de ser físicamente activo. Psicología y Salud, 17(2), 261-267. Recuperado de http://revistas.uv.mx/index.php/psicysalud/article/view/710/1249

Sáenz-López, P., Castillo, E., y Conde, C. (2009). Didáctica de la educación física escolar. Wanceulen E.F. Digital. 5. Recuperado de http://rabida.uhu.es/dspace/bitstream/handle/10272/3311/b15548764.pdf

Ministerio de Educación, Cultura y Deporte (2015). Encuesta de hábitos deportivos en España 2015, síntesis de resultados. Madrid: Secretaría General Técnica. Subdirección General de Documentación y Publicaciones. Recuperado de http://www.mecd.gob.es/servicios-al-ciudadano-mecd/dms/mecd/servicios-alciudadanomecd/estadisticas/deporte/ehd/Encuesta de Habitos Deportivos 2015 Sintesi $\underline{s \text { de Resultados.pdf }}$

\section{Agradecimientos}

Este artículo es fruto de mi Trabajo Fin de Grado (TFG) y me gustaría agradecer la ayuda de mi tutor, el Dr. Pedro Sáenz-López Buñuel, así como la colaboración de otros docentes e investigadores del Grupo de Investigación HUM-643. 